\title{
WDFB Digital Watermarking Method Based on Multiscale Geometrie Analysis
}

\author{
Guiming Shao ${ }^{a}$, Zhihua $\mathrm{Hu}^{\mathrm{b}}$ \\ College of Mathematical and computer Sciences, Huanggang normal University, \\ Hubei 438000, P. R.China \\ a $363818487 @ q q . c o m$, bhuzhihua123@126.com
}

\begin{abstract}
Keywords: Digital watermark; WDFB; watermark embedding; watermark extraction
\end{abstract}
\begin{abstract}
Combined with the human visual system and proposes a digital watermarking algorithm based WDFB domain. The algorithm is not the original image and watermark image WDFB coefficient directly superimposed, but the original image WDFB coefficient after pretreatment by the addition criteria to implement the embedded watermark. The experimental results show that the algorithm has strong robustness to shear, median filtering, noise and JPEG compression attacks. WDFB transform a new image analysis method is proposed for the lack of wavelet transform, but the watermarking method that there exist inadequacies; on the one hand, the need to use the original extract the watermark image should be along the zero-watermark the direction of the watermarking algorithm improvements.
\end{abstract}

\section{Introduction}

With the rapid development of multimedia technology, in order to solve the piracy problem of multimedia data, the digital watermarking technology came into being, and a high degree of attention, and has become an effective digital products copyright in recent years, robust image watermarking technology research andthe application to obtain protection and data authentication technology. Great progress, but to resist geometric attacks is still facing great difficulties, so is also one of the hotspots. At present, people study the anti-geometric attack watermarking technology mainly in the anti-rotation, zooming and panning (referred to as RST) connection, he proposed some algorithms. Literature [1] theoretically proved that the singular values of the images for geometric distortion (transpose, mirror, rotate, zoom, pan) invariance can be used to embedding watermark, but the algorithm is effective in resisting other attacks is not very ideals, such as noise attack and JPEG compression. Emi et al. [2] the discrete wavelet transform (DWT) and Singular Value Decomposition (SVD) combined, the singular value decomposition of the wavelet transform coefficients, were embedded watermark in four sub-bands, and achieved good results. . The mainstream algorithm is based on the transform domain watermark, there are discrete cosine transform domain (DCT) [3, 5] and discrete wavelet transform domain (DWT) [6] and so on. Image compression, image filtering and noise there is a certain resistance. DCT transformed image sub-block processing, and convenience is enhanced in the reconstructed image, the image will have a blocking effect. Wavelet transform because of its basis functions constructed isotropic and directional loss can not be effectively expressed the characteristics of high-dimensional functions.

\section{SWavelet Direction Filter Bank (WDFB)}

As we all know, the part of the dramatic changes in the image, such as edge, contour, and other details is an important feature of the image of local changes, it often contains more information than any other location in order to achieve a sparse representation of this information, WDFB transform is a kinds of wavelet transform based on a non-redundant multi-scale geometric analysis method, called wavelet directional filter banks (wavelet Direction Filter Bank, WDFB). a core issue of the directional filter banks is how the direction of Frequency Allocations to the desired accuracy, while maintaining the same number of samples. Latter can be down-sampling to achieve the progressive 
division of sub bands is the difficulty of the directional filter. two-dimensional directional filter banks Bamberger and Smith proposed DFB after the L layer decomposition can be divided into 21 different directions in the frequency domain, Figure 1.Bamberger construct directional filter banks, related to the modulation signal, as well as five individual plant sampling grid filters based on diamond-shaped filter group. In order to obtain the desired frequency domain is divided, you need a very complicated tree structure expansion, detail see [8]. In order to simplify the process of constructing the direction of the filter bank, refer to the literature $[4,9]$, through the sampling matrix and the equivalent translocation of the filter, combined with re-sampling the wrong cut, draw the concrete realization of the directional filter banks. Only consider the case of the analysis filter and synthesis filter can be similar to get.

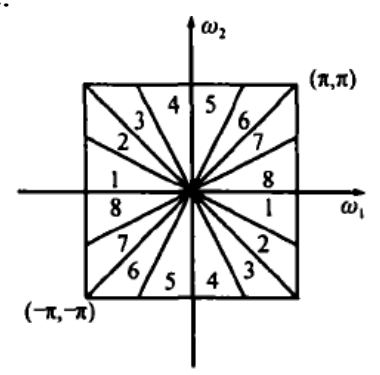

Fig.1 Eight-band directional partitioning

In multidimensional multirate systems, the sampling operation is defined on the grid, said that a $\mathrm{d}$-dimensional grid can be used a non-singular $\mathrm{d} \times \mathrm{d}$ integer matrix. For a fixed sampling grid, that is usually not only, The extract $\boldsymbol{M}$ of ${ }^{\boldsymbol{X}(\boldsymbol{n})}$ can be used the following formula:

$$
x_{d}[n]=x\left[M_{n}\right]
$$

Directional filter banks (DFB) to achieve multi-directional image decomposition, but it lacks the characteristics of multi-resolution and low-frequency component of the treatment effect on the image is not good low frequency component (DC component) and prone to leaks to several adjacentsubbands of the DFB itself is not the image representation. To this end, the wavelet decomposition and directional filter banks combining to provide a multi-resolution multi-directional image decomposition method, the WDF that is Contourlet transform thenon-redundant implementations can be more "sparse" image. specific implementation details, see [7].

\section{Algorithm Principle}

Compared with the classical wavelet transform, transform these areas in the image compression and demising WDFB show some advantages and potential, but also in the digital watermark, WDFB transformation has the following potential: multi-watermarking algorithm based on wavelet domain is to take the watermark information important coefficients of the transform coefficients to embed the original image on the low frequency wavelet coefficients, ignoring the high frequency there are still a number of important factors, low-frequency information is destroyed, the credibility of the watermark detection degree will decline; and WDFB transformation in D-wavelet transform based on the removal of a significant correlation between the high-frequency sub-band, will be evenly spread transform domain coefficients of the watermark information embedded in the transform domain data of the original image, regardless of experienced shear or compression attack, the similarity assessment compared with the wavelet transform has been improved to some extent.

\section{The watermark embedding and extraction model}

The basic idea of image watermarking systems literature [8] proposed a method, the method is embedded in a host image (original image), a secret information (watermark), and to ensure that the watermark is imperceptible and security, Shown in Figure 2. The digital image watermarking algorithm including the watermark embedding and watermark detection of the two processes. At the 
end of the watermark embedding, the original image to generate a watermark image after some adjustments, the embedded watermark image can then be demonstrated through a variety of media to the potential customer base, including images, may encounter a variety of attacks, including unintentional attacks and piracy by malicious attacks, and finally for the image of a copyright dispute can be verified to determine ownership of the copyright (usually requires a combination of the original image and watermark information).

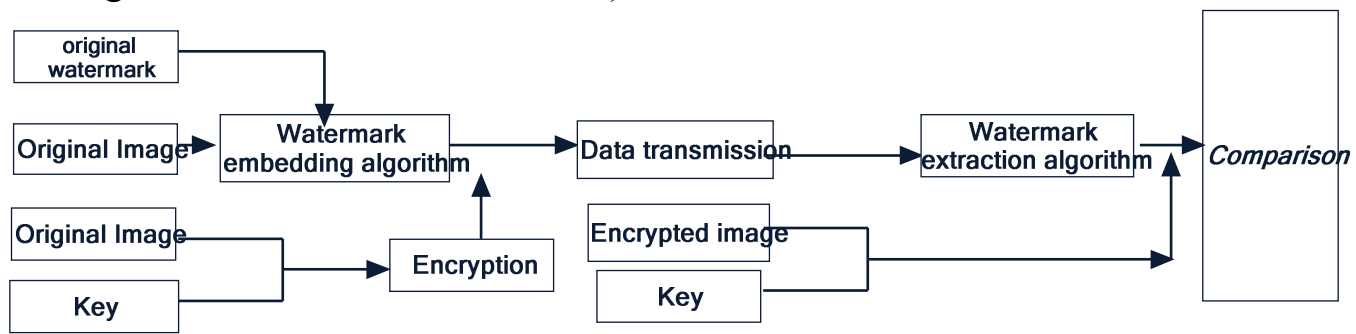

Fig.2 Watermark embedding and extraction model

Airspace watermark embedding method, the transform domain watermark embedding is usually achieved by modifying the frequency domain coefficients, usually the watermark embedding method of the addition criteria:

\section{Watermark Embedding Algorithm}

Watermark embedding process is described below:

1) Take the original image $\mathrm{M} \times \mathrm{M}$ and the watermark image $\mathrm{N} \times \mathrm{N}(\mathrm{M}=\mathrm{N} 2 \mathrm{P} P$ is a natural number);

2) watermark image transform WDFB will get WDFB coefficient divided by the coefficient of mean;

3) the original image WDFB transform all coefficient minus the mean of the watermark the image WDFB coefficient;

4) sort the coefficients obtained will corresponding watermark WDFB coefficient addition criteria and in the largest coefficient;

5) the use of the embedded watermark information after the original the image WDFB coefficients Bandelet inverse transform to get the image after the embedded watermark information.

\section{The watermark extraction algorithm}

The watermark extraction is about the public image of the watermark in the watermark information is separated from the. The watermark extraction process can be described as follows:

1) the watermarked image WDFB transform get corresponding WDFB coefficient;

2 ) in accordance with the inverse operation of addition criteria as well as the original image did pretreatment WDFB coefficient extracted to be detected is the watermark image.

\section{Simulation results and discussion}

The experiments take 512 pixels $\times 512$ pixels, the standard test image Lena original image, the watermark is a binary image of 64 pixels $\times 64$ pixels. The original image, the watermark image and the watermarked image without any treatment and removed watermark image below in Figure 2.

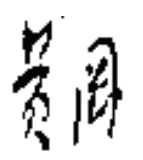

Fig.3(a) watermark

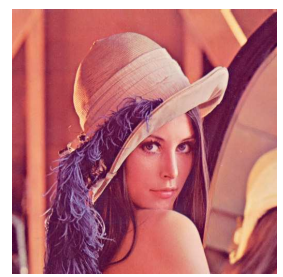

Fig.3(b) Lena

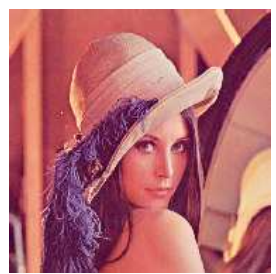

Fig.3(c) watermark image

Fig.3 Visual effects of the experiment 
It can be seen from Figure 2, the image embedded watermark information in comparison with the original image, there is no obvious distortion, it is difficult to see the difference. The same time, from the subjective visual point of view, to be perceived very well. Watermarked image and original image PSNR PSNR of $40.3586 \mathrm{~dB}$. The experiments show that the watermarked image without any image processing can be extracted watermark without distortion, the correlation coefficient NC 1 . To verify the robustness of the algorithm, the watermarked image were cut, and median filtering, noise, JPEG compression, and other conventional digital watermark attacks were compared with the original watermark image.

During transmission, the image is likely to be missing some information, in the case of retaining only part of the carrier image, the test can detect the presence of the watermark. 1/16, 1/4, 1/2 cut, the watermarked image results in Figure 4. Although the image is incomplete, but still can detect the watermark. Experimental results show that the algorithm of the watermark information to evenly distribute the original image, with a strong hidden. Several experimental results are as follows:

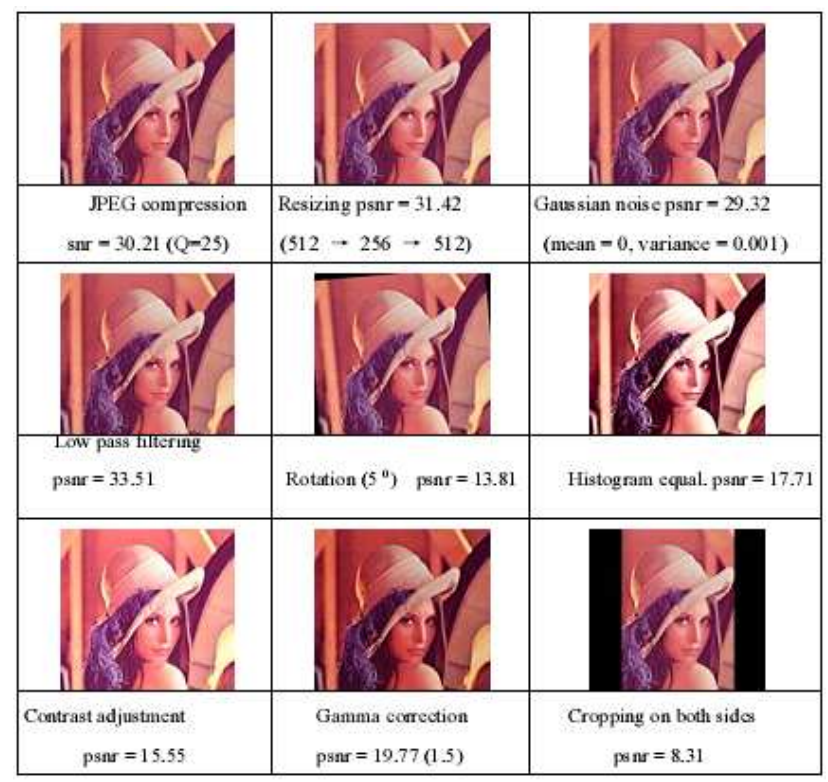

Fig.4 Attack renderings

Can also be seen from Figure 4, after the cut, add a watermark image information filtering, additive noise and JPEG compression, compared with wavelet transform of the similarity assessment have improved to varying degrees based on WDFB transform.

\section{Conclusions}

Combined with the human visual system is proposed based on the WDFB digital watermarking algorithm. The algorithm is not the original image and watermark image WDFB coefficient directly superimposed, but the original image WDFB coefficient after pretreatment by the addition criteria to implement the embedded watermark. The experimental results show that the algorithm has strong robustness to shear, median filtering, noise and JPEG compression attacks. WDFB transform a new image analysis method is proposed for the lack of wavelet transform, but the watermarking method that there exist inadequacies; on the one hand, the need to use the original extract the watermark image should be along the zero-watermark the direction of the watermarking algorithm improvements. On the other hand is hidden class algorithm and complicated to operate and extract information, to hide the amount of information can not be great, but its strong anti-attack capability, digital watermarking technology for copyright protection of digital works. 


\section{Acknowledgments}

This research was supported by the Hubei Province Natural Science Foundation 2010CDZ019, the outstanding young talents of the Hubei Provincial Department of Education project Foundation Q20122703.

\section{References}

[1] ZHOU B, CHEN J. A Geometric Distortion Resilient Image WatermarkingAlgorithm Based on SVD [J]. Image and Graphics, 2004, 9(4): 506 - 512.

[2] EMIR Ganic, AHMET M Eskicioglu. Robust DWT-SVD Domain Image Watermarking: Embedding Data In all frequencies [C] / /Proceeding soft he 2004 Workshop on Multimedia and Security. Magdeburg, Germany: ACM Special Interest Group onMultimedia, 2004: 166170.

[3] Cox I J, Kilian J, Leighton F T, et al. Secure spread spectrum watermarking for multimedia [ J]. IEEE Transaction on Image Processing, 1996, 6( 12): 1 673-1687.

[4] Cox I J, Kilian J, Leighton F T, et al. Secure spread spectrum watermarking for multimedia [R] . NEC Research Institute Technical Report, 1996, 11( 1) : 95-100.

[5] Cox I J, Kilian J, Leighton F T, et al. A secure, robust watermark for multimedia[J]. Information Hiding, 1996, 28( 2) :185-206.

[6] Xia X, Boncelet C G, Arce G R. Wavelet transform based watermark for digital images[J. Optics Express, 1998, 3 ( 12) :497-511.

[7] JIN Wei.A Novel Non - redundant Image Multiseale Geometrie Analysis, ACTA PHOTON ICA SINICA,2007,36(SUP)335-338.

[8] ZHU Xiu ge, NIU Hong juan, LI Deng feng . A digital image watermarking algorithm based on Bandelet transform,Journal of Fuzhou University(Natural Science Edition), 2011.39(05),686-692. 